\title{
Management of painful temporomandibular joint clicking with different intraoral devices and counseling: a controlled study
}

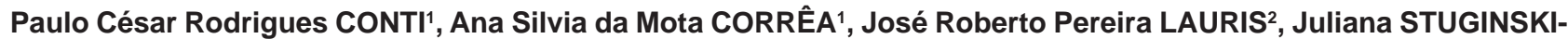 \\ BARBOSA $^{1}$
}

1- Department of Prosthodontics, Bauru School of Dentistry, University of São Paulo, Bauru, SP, Brazil.
2- Department of Department of Pediatric Dentistry, Orthodontics and Community Health, Bauru School of Dentistry, University of São Paulo, Bauru, SP, Brazil.

Corresponding address: Paulo César Rodrigues Conti - Departmento de Prótese - Faculdade de Odontologia de Bauru - Universidade de São Paulo - Al. Octavio Pinheiro Brisolla, 9-75 - 17012-901 - Bauru - SP - Brazil.

e-mail: pcconti@fob.usp.br

Submitted: November 4, 2014 - Modification: March 31, 2015 - Accepted: April 1, 2015

\section{ABSTRACT}

\begin{abstract}
$\mathrm{O}$ bjective: The benefit of the use of some intraoral devices in arthrogenous temporomandibular disorders (TMD) patients is still unknown. This study assessed the effectiveness of the partial use of intraoral devices and counseling in the management of patients with disc displacement with reduction (DDWR) and arthralgia. Material and Methods: A total of 60 DDWR and arthralgia patients were randomly divided into three groups: group I $(n=20)$ wore anterior repositioning occlusal splints (ARS); group II $(n=20)$ wore the Nociceptive Trigeminal Inhibition Clenching Suppression System devices (NTI-tss); and group III $(n=20)$ only received counseling for behavioral changes and self-care (the control group). The first two groups also received counseling. Follow-ups were performed after 2 weeks, 6 weeks and 3 months. In these sessions, patients were evaluated by means of a visual analogue scale, pressure pain threshold (PPT) of the temporomandibular joint (TMJ), maximum range of motion and TMJ sounds. Possible adverse effects were also recorded, such as discomfort while using the device and occlusal changes. The results were analyzed with ANOVA, Tukey's and Fisher Exact Test, with a significance level of 5\%. Results: Groups I and II showed improvement in pain intensity at the first follow-up. This progress was recorded only after 3 months in Group III. Group II showed an increase in joint sounds frequency. The PPT values, mandibular range of motion and the number of occlusal contacts did not change significantly. Conclusion: The simultaneous use of intraoral devices (partial time) plus behavioral modifications seems to produce a more rapid pain improvement in patients with painful DDWR. The use of NTI-tss could increase TMJ sounds. Although intraoral devices with additional counseling should be considered for the management of painful DDWR, dentists should be aware of the possible side effects of the intraoral device's design.
\end{abstract}

Keyw ords: Temporomandibular joint disorders. Temporomandibular joint disc. Arthralgia. Occlusal splints. Behavioral control.

\section{NTRODUCTION}

Temporomandibular disorders (TMD) embraces a group of musculoskeletal and neuromuscular conditions that involve the temporomandibular joint (TMJ), the masticatory muscles and all associated tissues ${ }^{5}$. Patients usually complain about both facial and TMJ pain, joint sounds and uncoordinated mandibular movement ${ }^{4}$. The anterior disc displacement is one of the most common TMJ internal derangements. It is a condition that can or be accompanied by pain and/or dysfunction or not ${ }^{5}$.

Treatment with intraoral appliances is often used to reduce pain and improve function in patients with most different subtypes of TMD. Diverse types of intraoral appliances have been used, and divergent theories have been proposed about their action mechanisms ${ }^{10}$. 
When displaced discs are part of the diagnosis, anterior repositioning splints can be used, designed to provide a guidance inclination to a therapeutic condyle-disc-fossa relationship in order to decrease TMJ pain and noise $3,4,14,19,20$. For many years, the use of this device was followed by extensive and irreversible occlusal reconstruction with the aim of perpetuating a "normal" condyle/disc relationship. Nowadays, although the maintenance of a normal disc/condyle relationship can be temporarily achieved, this is not the final treatment's intention, but is used to reduce pain severity. In general, clicking is not eliminated but its intensity could be reduced $^{20}$.

The Nociceptive Trigeminal Inhibition Clenching Suppression System (NTI-tss) device is also a form of intra-oral device and it was developed to produce an inhibitory stimulus, which is a reflex of the body to protect the teeth from excessive forces, preventing parafunctional habits. According to the manufacturer, NTI-tss allows for optimum musculoskeletal stablility (anterior-superior) in the condylar position. In the event the patient's condylar position is not optimal, the patient's condyle may re-position more posteriorly/superiorly during resolution of their symptoms. It could be used in patients with TMD, including those with TMJ and muscular pain, headaches or migraines.

Despite its use in clinical routines and in research ${ }^{1,17}$, the real benefits of the use of NTI-tss in arthrogenous TMD patients and the possible harm to dental occlusion or increased stimulus for pain and dysfunction in these patients are still unknown.

Moreover, counseling and behavioral changes can also be considered to be effective treatments and could be part of a conservative management for $\mathrm{TMD}^{1,21}$. Based on this, this study is aimed at assessing the effectiveness of counseling therapies for behavioral changes, with or without the partial use of intraoral devices (anterior repositioning splint or NTI-tss) in a 3 months period. The null hypothesis is that there is no difference between groups for $\mathrm{TMJ}$ pain reduction after this period.

\section{MATERI AL AND METHODS}

This study was a controlled randomized clinical trial, involving individuals with signs of disc displacement and TMJ pain, designed to test the efficacy of intraoral devices and counseling in the control of TMJ pain.

\section{Sample}

Patients were select from those attending the Orofacial Pain Clinic. Sixty patients were enrolled according to the following criteria:

Inclusion criteria:

Adults aged 18 years or more;
Report of pain in preauricular region, in the last 30 days, worsened by functional activities, such as chewing and talking;

Presence of disc displacement with reduction (IIa) and arthralgia (IIIa) according to the Research Diagnostic Criteria for Temporomandibular Disorders (RDC-TMD) ${ }^{6}$.

Exclusion criteria:

Individuals with a recent history of trauma in the face and/or neck area;

Individuals with systemic diseases that can affect TMJ;

History of TMJ surgery;

Individuals with dental pain;

Individuals with myofascial pain, disc displacement without reduction or osteoarthritis according to the RDC-TMD;

Individuals under dental or TMD management; Individuals wearing full or partial dentures;

Individuals with major psychological disorders.

\section{Experimental procedure}

After receiving informed consent from all subjects who met the initial criteria, an assistant randomly assigned the participants into one of the three groups as described below:

Group I (anterior repositioning occlusal splint and counseling $-n=20$ ): patients received therapy with an anterior repositioning occlusal splint and counseling for habits and behavioral changes ${ }^{21}$. The splints were built with rigid acrylic for wearing in the upper arch. The degree of protusion was the minimum necessary to eliminate joint clicking and the splints were adjusted to allow for a proper occlusal relationship, with contacts distributed well over the occlusal surface. Patients were instructed to wear their splints only while sleeping. The behavioral counseling included instructions containing information about relaxation techniques, sleep hygiene, diet modification, hot thermotherapy, as well as avoidance of caffeine and awaking clenching ${ }^{1,21}$.

Group II (NTI-tss and counseling $-\mathrm{n}=20$ ): patients received therapy with NTI-tss device and counseling for habits and behavioral changes. The NTI-tss devices were adjusted according to the manufacturer's instructions. This device covered only the upper incisors with a single contact with the mandibular incisors. Instructions were given for sleep-time use only, as in group I. The same counseling instructions were given to patients in this group.

Group III (control, counseling only - $n=20$ ): patients received only the previously described instructions about counseling for behavioral changes.

Two experienced examiners, aligned to perform all necessary RDC/TMD evaluations, appliance delivery, 
behavioral counseling and patient follow-ups. The first examiner handled the randomization process and provided the proper therapy, whereas the second one performed the follow-up examinations and collected all of the clinical data, using a "blind" design with no knowledge of the individual's group.

The follow-up included three visits after the treatment started: at 2 weeks, 6 weeks and 3 months. In each visit the following variables were analyzed:

At the initial and follow-up visits the individuals independently rated the current pain intensity (PI) on a $100-\mathrm{mm}$ line by marking a point on the line between the two extremes where the left extreme of the scale is marked "no pain" and the right one is marked "the worst imaginable pain". The distance from the start line to the point at which the line was marked was measured with a ruler to determine the pain intensity ${ }^{18}$.

PPT was performed using a digital pressure algometer with a $1 \mathrm{~cm}^{2}$ flat-circular shape tip at one end (Mod. DDK-20, Kratos Equipamentos Industriais, Cotia, SP, Brazil) once at each TMJ. Before the assessment, the TMJ lateral poles were located by asking the patient to open and close their mouth three times with the operator's finger placed in front of the tragus. Before PPT measurements, each patient undertook a short training session for familiarization with the algometer, its handheld device and its application method. The probe was then held perpendicularly at the lateral pole of the TMJ to apply the pressure over the TMJ. The pressure application rate was approximately $0.5 \mathrm{Kgf} / \mathrm{cm}^{2} / \mathrm{sec}$. During the examination, the operator's hand passively supported the individual's head. The participant was asked to indicate when the sensation changed from pressure to pain by pushing a button, part of the device used in the present investigation.

Patients were requested to open the mouth to the maximum. A measurement was made with a flexible ruler between the top and bottom edges, taking as reference the midline. In the presence of pain the patient was asked to open his mouth until present painful sensation. The value of the maximum mouth opening was always corrected by adding the overbite.

The presence of TMJ sounds was performed according to an RDC/TMD examination ${ }^{5}$. Fingers were placed over the subject's TMJs (preauricular area), anterior to the tragus of the ear. The subject was asked to slowly open their mouth as wide as possible 3 times. Each closure should bring the teeth completely together to maximum intercuspation. TMJ sounds (clicking) on opening or closing as detected by palpation were recorded.

The number of occlusal contacts between the upper and lower teeth was recorded using a ribbon and a Miller clamp, with the patient was seated in an upper body position and with dried teeth ${ }^{1}$.

The patients of groups I and II were asked about their comfort when the splint adjustment was performed. For maintaining the blinded strategy, patients were asked to complete a form with the following options for splint comfort: "maintained", "changed for the better", "changed for the worse" or "don't know".

In each visit, a comprehensive assessment of splint adjustments was performed for Group I and II and the counseling and behavioral changes information were reinforced in all groups.

\section{Data analysis}

Analysis of variance (ANOVA) followed by post hoc Tukey was used for comparing age, pain intensity, PPT, maximum opening mouth and number of occlusal contacts inter and intra-group, as these variables followed the normal distribution. The Fisher exact test compared the presence of TMJ sounds (clicking) and the evaluation's of splint comfort.

Significance was established at the level of $5 \%$, with CI of $95 \%$.

\section{RESULTS}

From 300 potential participants, 60 (20\%) fulfilled the initial criteria and agreed to participate. At the beginning these 60 patients (58 female and 2 male) were divided into three groups. Each group had 20 participants. After 3 months, 33 patients completed the treatment (Figure 1). Twenty-seven patients dropped out because of the lack of interest or difficulties in returning to the University for follow-up visits. These individuals were, therefore, excluded from the final analysis.

The mean age of the sample was 38.35 years of age for group I, 38.4 years of age for group II and 46 years of age for group III ( $p>0.05)$.

A significant decrease in pain intensity was found for all groups when the VAS alterations between the initial and 3 month evaluations were considered (intragroup analysis) $(p<0.05)$ (Figure 2 ). However, this fact occurred earlier in groups I and II ( 2 weeks) and only in the final evaluation, at 3 months, for group III. In inter-group analysis, anterior repositioning of the occlusal splint and counseling for behavioral changes (group I) were able to significantly reduce pain intensity when compared with the counseling and behavioral therapy only (group III) in the 6 week ( $p-0.003$ ) and 3 month $(p=0.01)$ follow-up evaluations.

After 3 months, the percentage of patients responsive to treatment was $83.3 \%$ for group I, $75 \%$ for group II and $55.5 \%$ for group III.

There were no differences between the PPT 
values, mandibular range of motion and the number of occlusal contacts within or between groups at different times in the right or left TMJ (Table 1).

In the beginning of the study, all patients had a TMJ click at least in one side. When joint sounds (clicking) were investigated after 3 months, a decrease in frequency for groups I and III was observed. On the other hand, an increase in frequency for those in group II (Figure 3) was detected, although with no significance.

After 2 weeks of splint use, patients wearing anterior repositioning occlusal splints reported themselves as being more comfortable and having their initial condition improved $(100 \%)$ compared to those in group II $(66.7 \%)(p<0.05)$.

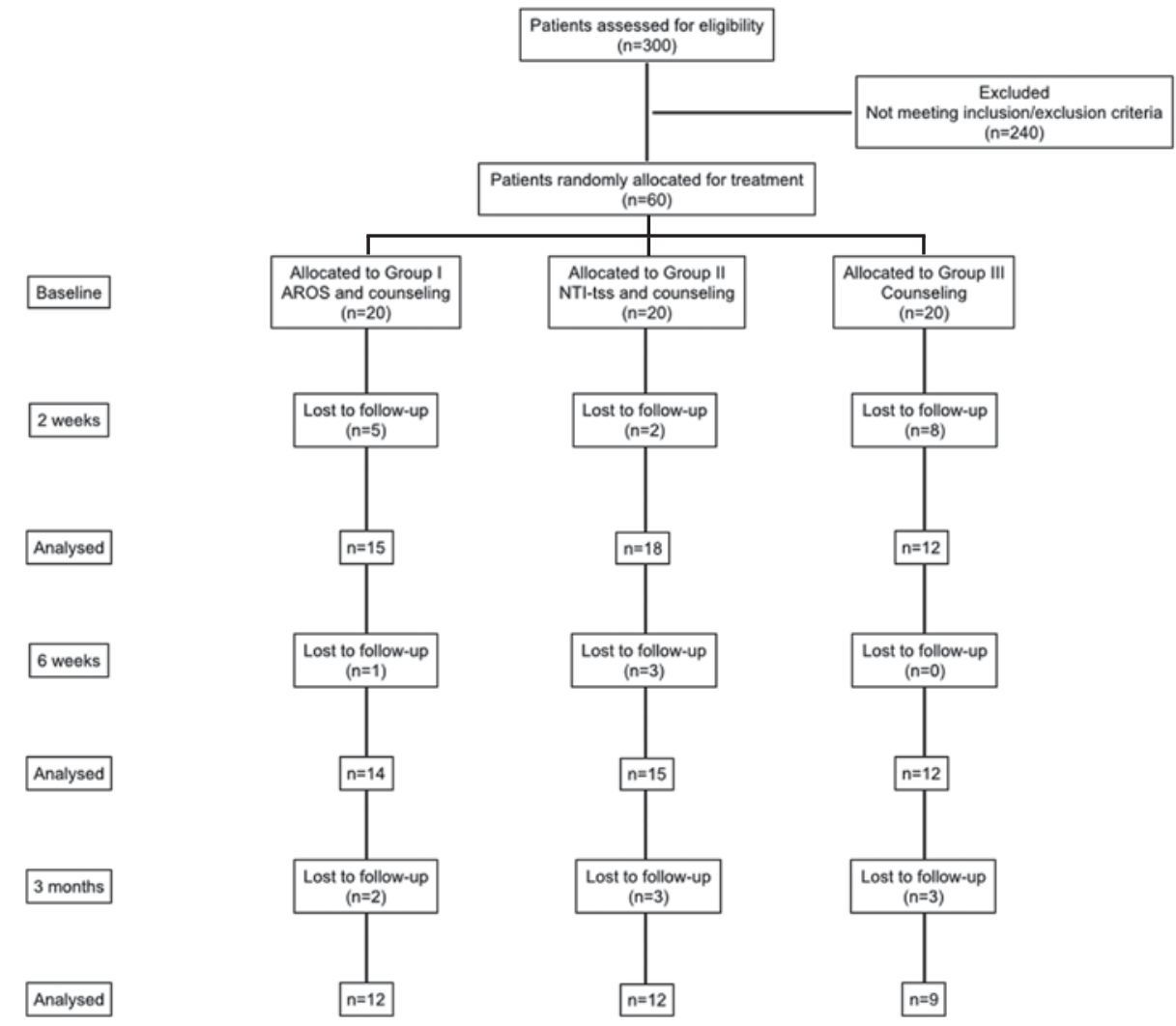

Figure 1- Three hundred patients were clinically examined according to the inclusion and exclusion criteria. Screening showed that 240 subjects met one or more of the exclusion criteria. The remaining 60 patients were randomized for treatment in the study. AROS = anterior repositioning occlusal splint; NTI-tss = Nociceptive Trigeminal Inhibition Clenching Suppression System

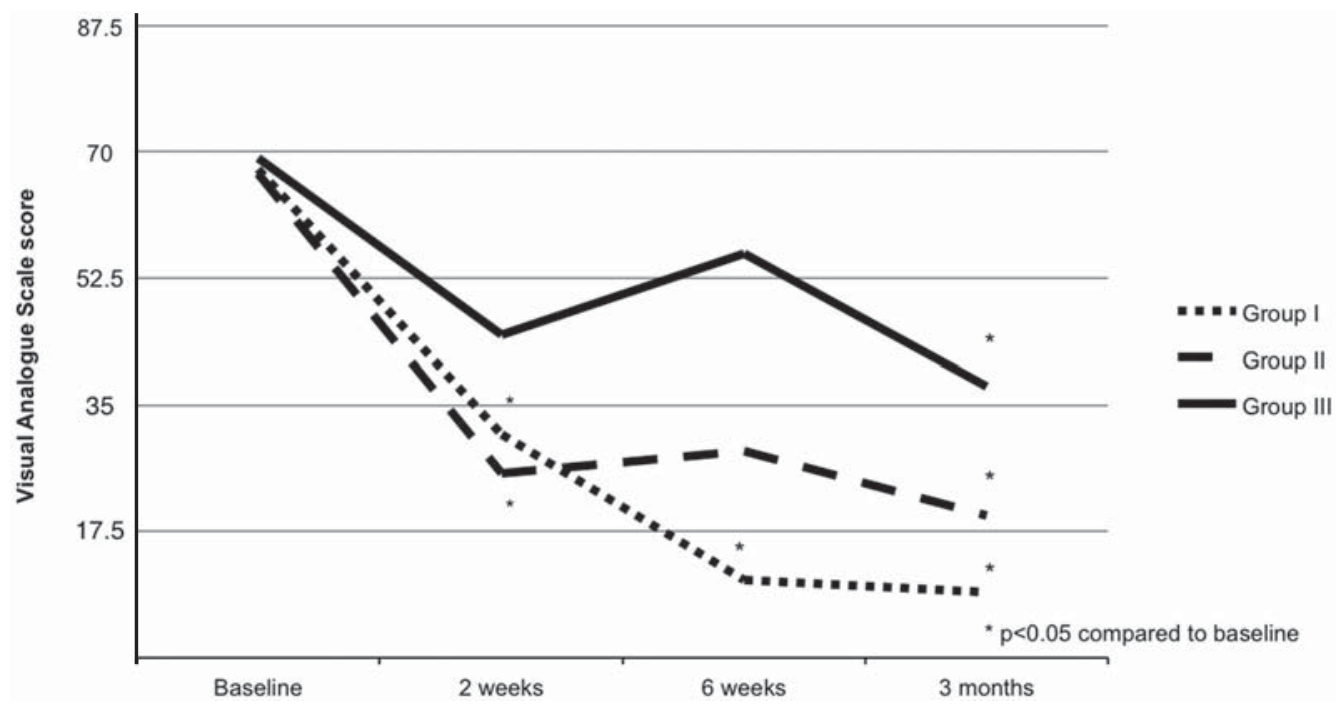

Figure 2- Pain intensity for the three groups at different times with intra-group ANOVA analysis test 
Table 1- The mean standard deviation Visual Analogue Scale (VAS) score, pressure pain threshold (kgf/ $\left.\mathrm{cm}^{2}\right)$ and mandibular range of motion (in millimeters) for all groups at baseline and 3 months (ANOVA and Tukey's test)

\begin{tabular}{ccccccccc}
\hline & \multicolumn{2}{c}{ Group I } & \multicolumn{2}{c}{ Group II } & \multicolumn{2}{c}{ Group III } & \multicolumn{2}{c}{$\begin{array}{c}\text { p-values between } \\
\text { groups }\end{array}$} \\
& Baseline & 3 Months & Baseline & 3 Months & Baseline & 3 Months & Baseline & 3 Months \\
\hline & & & \multicolumn{2}{c}{ Pressure Pain Threshold } \\
\hline TMJ right & $1.67(0.47)$ & $1.58(0.33)$ & $1.76(0.71)$ & $1.61(0.49)$ & $1.34(0.35)$ & $1.30(0.29)$ & 0.68 & 0.16 \\
\hline TMJ left & $1.57(0.33)$ & $1.53(0.44)$ & $1.75(0.65)$ & $1.63(0.57)$ & $1.37(0.51)$ & $1.47(0.39)$ & 0.67 & 0.74 \\
\hline $\begin{array}{c}\text { Mandibular } \\
\text { range of } \\
\text { motion }\end{array}$ & $40.5(10.2)$ & $41.2(7.2)$ & $38.4(7.9)$ & $42.2(6.4)$ & $39.8(6.9)$ & $39.3(6.1)$ & 0.45 & 0.61 \\
\hline $\begin{array}{c}\text { Number of } \\
\text { occlusal } \\
\text { contacts }\end{array}$ & $25.7(8.6)$ & $26.7(7.2)$ & $26.6(11.3)$ & $26.9(9.1)$ & $23.7(6.2)$ & $22.9(4.4)$ & 0.95 & 0.45 \\
\hline
\end{tabular}

TMJ=Temporomandibular joint

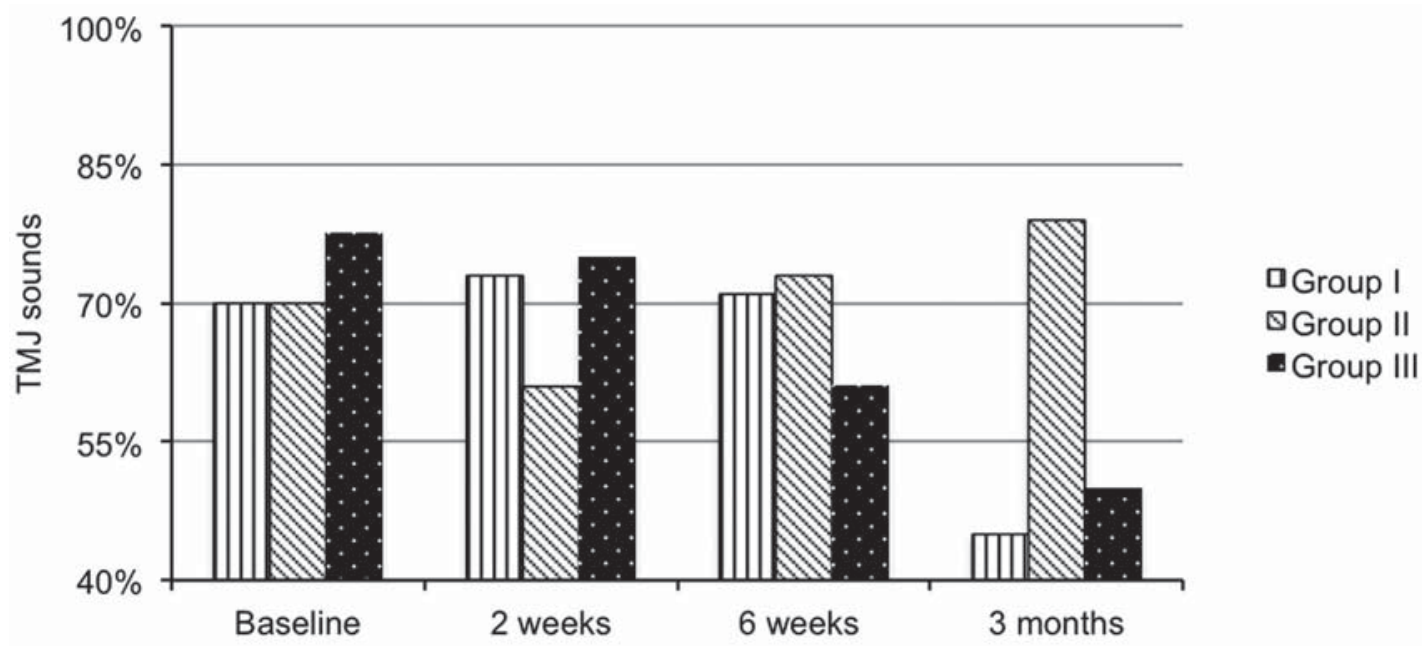

Figure 3- Presence of temporomandibular joint (TMJ) clicking sounds for the three groups at different times. For each subject, two TMJs were considered $(n=120)$

\section{SCUSSI ON}

This study was aimed at evaluating the effectiveness of different intraoral devices associated with counseling for behavioral changes in the management of TMJ pain and dysfunction in a short-term evaluation. Our most important findings were: [1] All management strategies provided a significant effect in ameliorating the reported pain intensity; [2] patients wearing occlusal devices accompanied by counseling and behavioral changes reported faster significant improvements, when compared to the control group, with no splints, highlighting the importance of the intraoral device in the management of TMJ pain; [3] there were no TMJ sound (clicking) improvements in patients wearing NTI-tss splint. Based on that, the null hypothesis is rejected.

The within-group analysis demonstrated a significant decrease in VAS values for group I and II after 2 weeks, when compared to baseline values, whereas the same occurred only after 3 months for group III.

Anterior repositioning appliances are used as part of management strategies for TMJ internal derangements. The improvement for splint therapy groups, found here, agree with several studies $^{3,13,14,20,22}$. The reasons for this fact could be attributed to different mechanisms. The partial maintenance of a normal disc/condyle relationship (only at night time) and/or reduced joint pressure, which could decrease overload to retrodiscal tissues, allowing for healing and adaptive changes to occur is probably an important occurrence in this scenario ${ }^{3}$. The fact that patients wearing the splints only on a part-time basis allowed for the "contact" between the condyle and retrodiscal structures during normal function, which can help to 
stimulate the fibrosis witnessed and the formation of a "pseudodisc", providing protection to structures previously damaged. It is a different strategy from those who perpetuate this "therapeutic" position with irreversible treatment, as had been suggested in the past.

Other action mechanisms could explain the earlier progress for those wearing occlusal devices, such as the presence of a new intraoral condition, which are designed to interfere with the impact of certain trigeminal neural circuits ${ }^{10}$, somehow affected the overall pain perception. A recent study with NTI-tss devices and a stabilization splint for muscular TMD management reported similar results ${ }^{1}$.

The improvement for the control group certainly illustrates the benign aspect of these conditions and may be a warning about the need for irreversible procedures $^{3}$. In fact, a recent 2 - to -3 year followup study showed that the percentage of patients with arthralgia decreased almost $53 \%$ without management, which agrees with the favorable natural course of the condition ${ }^{16}$.

Not only the natural evolution of the disease, but also placebo effects and also the regression towards the mean may have played an important role in the effectiveness of the different modalities for TMJ pain management.

Besides the change in pain intensity, no significant differences were found for PPT values between groups. PPT, as well VAS, are subjective clinical measurement tools and can only approximate a true measurement of pain ${ }^{9}$. Pain has been accepted as a sensation, influenced by several aspects, so it is difficult to determine how much of the reported pain is a result of a localized stimulation of the injured site, impairment of peripheral or central pain pathways or due to some emotional component ${ }^{2}$. Moreover, it is possible that the placement of the algometer did not directly stress the injured tissues in TMJ cases, despite being within the area of self-reported pain ${ }^{9}$, which could explain the actual results. It could be expected that alterations in the PPT values could occur in a long-term analysis ${ }^{1}$, which unfortunately we were unable to determine in this investigation.

As mentioned above, the use of repositioning splints in the present study did not define objective permanent disc recapturing. The exact disc position before and after management could not be established as a magnetic resonance image (MRI) and was not a part of the methodology. The resolution of clicking is probably due to morphological alterations in the disc itself, which could eliminate the physical barrier for jaw translation and thus decrease the sound ${ }^{4}$. In this study, the resolution of TMJ clicking was not the primary objective as splints are no longer used for this purpose ${ }^{3,4}$. Longitudinal observations have shown that few patients with disc displacement with reduction go through the staged phases of anteriorization that could end in disc displacement without reduction ${ }^{11,16}$. A natural and progressive adaptation of TMJ structures to loading may explain the general improvement of clicking, regardless if they wore an anterior repositioning splint or not ${ }^{3}$.

Only the group that wore NTI-tss device showed an increased frequency of TMJ clicking sounds. Part of the occlusal force applied on the teeth is transmitted to the TMJ and the more anterior the teeth contact, the higher the load over the temporomandibular joint per unit of bite force ${ }^{12}$. As NTI-tss design does not permit contact in the posterior region, its use may have increased TMJ loading over time?. As a consequence, the remodeling process of the disc may have been compromised in this group. The initial discomfort in using the device could also explain these findings.

Full-time use of anterior positioning devices has frequently been associated with posterior open bite, occlusal alterations and muscle contracture of the lateral pterygoid ${ }^{19}$. Conti, et al. ${ }^{3}$ (2005), however, showed in a long-term study, that partial use of the devices did not significantly change the number of occlusal contacts or cause any skeletal problems, which was also was found in this study. Although only devices that change the maxilo-mandibular relationship were tested in this investigation, one should be advised that improvements in pain and dysfunction have also been reported with the use of a "regular" stabilization, flat splint, with no changes in mandibular positioning ${ }^{3}$. This modality could be a good, reliable and safe option to manage those patients.

Adverse effects can also occur with NTI-tss use. Occlusal changes were reported in previous studies after 3 to 6 months ${ }^{15}$, as well as aspiration, swallowing and tooth mobility ${ }^{8,17}$. In this study, no occlusal alterations or adverse effects were found. However, as discuss above, patients had reported discomfort in using NTI-tss during the first 15 days.

The low number of individuals in the final follow-up, especially in the control group, and the short-term nature of the evaluation must be considered when judging our results. Over time, $45 \%$ of patients dropped out. The use of a intraoral device may have motivated the patient to not miss appointments as the patient's feeling of being "under treatment" is an important influence in maintaining interest as the number of patients that dropped out was lower in groups I and II. Moreover, the patients' treatment expectations about the behavioral counseling are unknown and could have influenced the treatment's adherence and outcomes and also the dropout rate.

As a significant improvement, it had also been 
obtained that with counseling and behavioral strategies only, the use this therapy is suggested to be part of a conservative management for arthrogenous TMD.

\section{CONCLUSI ON}

Occlusal devices such as the anterior repositioning occlusal splint and NTI-tss or behavioral changes are effective in the management of the pain associated with disc displacement with reduction. The simultaneous use of occlusal devices appears to produce a faster improvement. The use of NTI-tss could increase TMJ sounds (noise) and its effectiveness and safety are still to be proven in long-term studies.

\section{REFERENCES}

1- Conti PC, Alencar EN, Mota Corrêa AS, Lauris JR, Porporatti AL, Costa YM. Behavioural changes and occlusal splints are effective in the management of masticatory myofascial pain: a short-term evaluation. J Oral Rehabil. 2012;39(10):754-60.

2- Conti PC, Azevedo LR, Souza NV, Ferreira FV. Pain measurement in TMD patients: evaluation of precision and sensitivity of different scales. J Oral Rehabil. 2001;28(6):534-9.

3- Conti PC, Miranda JE, Conti AC, Pegoraro LF, Araújo CR. Partial time use of anterior repositioning splints in the management of TMJ pain and dysfunction: a one-year controlled study. J Appl Oral Sci. 2005;13(4):345-50.

4- Conti PC, Santos CN, Kogawa EM, Castro Ferreira Conti AC, Araujo CR. The treatment of painful temporomandibular joint clicking with oral splints: a randomized clinical trial. J Am Dent Assoc. 2006;137(8):1108-14.

5- De Leeuw R, Klasser GD, eds. Orofacial pain: guidelines for assessment, diagnosis, and management. Chicago: Quintessence Publishing Co; 2013. 312 p.

6- Dworkin SF, LeResche L. Research diagnostic criteria for temporomandibular disorders: review, criteria, examinations and specifications, critique. J Craniomandib Disord. 1992;6(4):301-55. 7- Israel HA. Current concepts in the surgical management of temporomandibular joint disorders. J Oral Maxillofac Surg. 1994;52(3):289-94.

8- Jokstad A, Mo A, Krogstad BS. Clinical comparison between two different splint designs for temporomandibular disorder therapy. Acta Odontol Scand. 2005;63(4):218-26.

9- Kamper SJ, Maher CG, Hush JM, Pedler A, Sterling M. Relationship between pressure pain thresholds and pain ratings in patients with whiplash-associated disorders. Clin J Pain. $2011 ; 27(6): 495-501$.
10- Klasser GD, Greene CS. Oral appliances in the management of temporomandibular disorders. Oral Surg Oral Med Oral Pathol Oral Radiol Endod. 2009;107(2):212-23.

11- Könönen M, Waltimo A, Nyström M. Does clicking in adolescence lead to painful temporomandibular joint locking? Lancet. 1996;347(9008):1080-1.

12- Koolstra JH, van Eijden TM, Weijs WA, Naeije M. A threedimensional mathematical model of the human masticatory system predicting maximum possible bite forces. J Biomech. 1988;21(7):563-76.

13- Kurita H, Ohtsuka A, Kurashina K, Kopp S. A study of factors for successful splint capture of anteriorly displaced temporomandibular joint disc with disc repositioning appliance. J Oral Rehabil. 2001;28(7):651-7.

14- Madani AS, Mirmortazavi A. Comparison of three treatment options for painful temporomandibular joint clicking. J Oral Sci. 2011;53(3):349-54.

15- Magnusson T, Adiels AM, Nilsson HL, Helkimo M. Treatment effect on signs and symptoms of temporomandibular disorders comparison between stabilisation splint and a new type of splint (NTI). A pilot study. Swed Dent J. 2004;28(1):11-20.

16- Manfredini D, Favero L, Gregorini G, Cocilovo F, Guarda-Nardini L. Natural course of temporomandibular disorders with low painrelated impairment: a 2-to-3-year follow-up study. J Oral Rehabil. 2013;40(6):436-42.

17- Stapelmann H, Türp JC. The NTI-tss device for the therapy of bruxism, temporomandibular disorders, and headache - where do we stand? A qualitative systematic review of the literature. BMC Oral Health. 2008;8:22.

18- Svensson P, Arendt-Nielsen L, Nielsen H, Larsen JK. Effect of chronic and experimental jaw muscle pain on pain-pressure thresholds and stimulus-response curves. J Orofac Pain. 1995;9(4):347-56.

19- Tallents RH, Katzberg RW, Macher DJ, Roberts CA. Use of protrusive splint therapy in anterior disk displacement of the temporomandibular joint: a 1 - to 3-year follow-up. J Prosthet Dent. 1990;63(3):336-41.

20- Tecco S, Festa F, Salini V, Epifania E, D'Attilio M. Treatment of joint pain and joint noises associated with a recent TMJ internal derangement: a comparison of an anterior repositioning splint, a full-arch maxillary stabilization splint, and an untreated control group. Cranio. 2004;22(3):209-19

21- Truelove E, Huggins $\mathrm{KH}$, Mancl L, Dworkin SF. The efficacy of traditional, low-cost and nonsplint therapies for temporomandibular disorder: a randomized controlled trial. J Am Dent Assoc. 2006;137(8):1099-107.

22- Williamson EH. Temporomandibular dysfunction and repositioning splint therapy. Prog Orthod. 2005;6(2):206-13. 